\title{
Biogeografia do gênero Mikania Willd. (Asteraceae) no Rio Grande do Sul, Brasil ${ }^{1}$
}

\author{
Mara Rejane Ritter ${ }^{2}$ e Jorge Luiz Waechter ${ }^{2}$
}

Recebido em 03/06/2003. Aceito em 18/02/2004

\begin{abstract}
RESUMO - (Biogeografia do gênero Mikania Willd. (Asteraceae) no Rio Grande do Sul, Brasil). A distribuição geográfica das 39 espécies nativas de Mikania Willd. no Rio Grande do Sul foi estudada em duas escalas: uma escala regional, utilizando regiões fisiográficas como unidades, e uma escala continental, considerando regiões político-geográficas como unidades. A riqueza específica e a amplitude de distribuição foram obtidas diretamente de matrizes de presença e ausência, relacionando espécies e regiões. A matriz regional foi analisada por dois métodos multivariados, uma análise de agrupamentos e uma análise de coordenadas principais, utilizando o complemento do coeficiente de Jaccard como elemento de comparação (dissimilaridade). Na escala regional as regiões mais ricas foram o Litoral e a Depressão Central, enquanto na escala continental, a maioria das espécies sul-rio-grandenses foram também encontradas no Sudeste do Brasil e no Sul da América do Sul. A análise multivariada evidenciou dois grupos principais no Estado, um oriental e outro ocidental. Em cada um desses grupos foram evidenciados dois subgrupos relacionados a características climáticas e geológicas do Estado. Os padrões de distribuição de Mikania confirmam o caráter tropical do gênero e as principais rotas de migração descritas para a flora do Sul do Brasil.
\end{abstract}

Palavras-chave: Mikania, Asteraceae, biogeografia, Rio Grande do Sul

\begin{abstract}
Biogeography of the genus Mikania Willd. (Asteraceae) in Rio Grande do Sul, Brazil). The geographical distribution of the 39 species of Mikania Willd. native in Rio Grande do Sul was studied at two scales: a state-regional scale, using physiographic regions as units, and an american-continental scale, considering politic-geographic regions as units. Specific richness and distribution amplitude were obtained directly from presence and absence matrixes, relating species and regions. The state-regional matrix was also analysed by two multivariate methods, a cluster analysis and a principal coordinate analysis, using the complement of the Jaccard coefficient as a measure of dissimilarity. At the state-regional scale, the richest regions were the Coastal Plain and the Central Depression, whereas at the american-continental scale, most of the South Riograndean species were also found in Southeastern Brazil and Southern South America. The multivariate analysis evidenced two main groups in Rio Grande do Sul, one eastern and another western. Within each of these groups, two subgroups related to climatic and geological features were evidenced. The distribution patterns of Mikania confirm the tropical character of the genus, and the main migration routes described for the southern Brazilian vascular flora.
\end{abstract}

Key words: Mikania, Asteraceae, biogeography, Rio Grande do Sul

\section{Introdução}

O gênero Mikania Willd. apresenta cerca de 450 espécies (Holmes 1996). É um gênero com distribuição pantropical, mas com extensões nas áreas temperadas da América e sudeste da África. Para o Brasil são citadas cerca de 171 espécies (King \& Robinson 1987). No Rio Grande do Sul constatou-se a ocorrência de 39 espécies. Há dois grandes centros de diversidade do gênero na América do Sul. O primeiro, com aproximadamente 170 espécies (cerca de 150 endêmicas), localiza-se desde Minas Gerais e Rio de Janeiro até o Paraná e Santa Catarina, com muitas espécies estendendo-se até o Paraguai, Uruguai e
Argentina. O segundo, com aproximadamente 150 espécies (cerca de 130 endêmicas), localiza-se nos países andinos, da Colômbia até a Bolívia (Holmes 1995). Fora destes centros de diversidade, o número de espécies é reduzido. A maioria das espécies de Mikania encontra-se distribuída na América do Sul, sendo um dos poucos gêneros de Asteraceae que alcançaram sucesso nas terras baixas da região Amazônica, área considerada inadequada para a maioria dos membros da família. De acordo com Holmes (1995), o hábito trepador foi importante para a grande representatividade do gênero nesta região, ocupando principalmente áreas úmidas de bordas de rios e lagos. As espécies eretas estão adaptadas ao

\footnotetext{
Parte da Tese de Doutorado do primeiro Autor

2 Departamento de Botânica, Instituto de Biociências, Universidade Federal do Rio Grande do Sul, Av. Bento Gonçalves, 9500 - Bloco IV, CEP 91509-900, Campus do Vale, Porto Alegre, RS, Brasil

3 Autor para correspondência: mrritter@terra.com.br
} 
ambiente mais seco de savanas, cerrados e campos. O hábito predominante é o volúvel, mas ocorrem plantas apoiantes, decumbentes e eretas. No Rio Grande do Sul, as espécies eretas e decumbentes de Mikania são campestres, enquanto que as volúveis e apoiantes habitam preferencialmente bordas e interior de matas.

Segundo Stuessy \& Garver (1996), o papel adaptativo do pappus na dispersão dos frutos contribui para o sucesso reprodutivo da família. Os frutos são dispersos principalmente pelo vento, mas também pela água e por animais (Holmes 1995). Em Mikania a eficiente dispersão dos frutos pelo vento, sem dúvida, é um dos aspectos responsáveis pela ampla distribuição do gênero por diferentes regiões. King \& Robinson (1987) mencionaram a dispersão de frutos de Mikania a longa distância por pássaros, uma vez que parece lógico que um gênero notável por sua invasão ao hábitat de pássaros, as árvores, tenha aumentada a oportunidade de ter seus frutos transportados por estes animais.

Dois importantes estudos biogeográficos da família Asteraceae para o Sul do Brasil foram apresentados por Rambo (1952) e por Mondin \& Baptista (1996). No primeiro trabalho, Rambo (l.c.) discutiu a distribuição de espécies setentrionais e meridionais, abrangendo, respectivamente, os táxons com centro de dispersão nas regiões tropicais e os táxons com centro de dispersão nas regiões temperadas da América do Sul. Já o artigo de Mondin \& Baptista (l.c.) discutiu as relações biogeográficas da tribo Mutisieae sensu Cabrera no Rio Grande do Sul, evidenciando a existência de dois grupos principais, coincidentes com unidades biogeográficas baseadas em parâmetros florístico-vegetacionais.

Estudos fitogeográficos em grupos tropicais e temperados ocorrentes no Rio Grande do Sul são encontrados em famílias predominantemente campestres, como Fabaceae (Rambo 1953; Miotto \& Waechter 1996) e Poaceae (Longhi-Wagner 1990; Longhi-Wagner \& Zanin 1998; Boechat \& LonghiWagner 2000), além de famílias predominantemente florestais, como Orchidaceae (Waechter 1998).

O presente trabalho tem por objetivo verificar os padrões de distribuição das espécies de Mikania ocorrentes no Rio Grande do Sul, no âmbito do Estado e no âmbito da América.

\section{Material e métodos}

A distribuição das espécies de Mikania baseia-se em ampla revisão de herbários e de coletas originais e fazem parte da revisão do gênero recentemente concluído pelo primeiro Autor. As informações sobre as 39 espécies de Mikania encontram-se na Tab. 1.

Foram elaboradas duas matrizes de dados, uma relacionando as espécies com as regiões fisiográficas do Rio Grande do Sul, e outra, com as regiões políticogeográficas da América.

Tabela 1. Espécies de Mikania Willd. ocorrentes no Rio Grande do Sul, agrupadas de acordo com o hábito e o hábitat: BA - banhado; $\mathrm{BM}$ - beira de mata; CA - campo; IM - interior de mata.

\begin{tabular}{|c|c|}
\hline Hábito/Espécie & Hábitat \\
\hline \multicolumn{2}{|l|}{ Decumbente } \\
\hline M. decumbens Malme & $\mathrm{CA}$ \\
\hline \multicolumn{2}{|l|}{ Ereto } \\
\hline M. anethifolia (DC.) Matzenbacher & $\mathrm{CA}$ \\
\hline M. fulva (Hook. \& Arn.) Baker & $\mathrm{CA}$ \\
\hline M. oblongifolia DC. & $\mathrm{CA}$ \\
\hline M. pinnatiloba $\mathrm{DC}$. & $\mathrm{CA}$ \\
\hline M. thapsoides DC. & $\mathrm{CA}$ \\
\hline M. viminea $\mathrm{DC}$. & $\mathrm{BA}, \mathrm{CA}$ \\
\hline \multicolumn{2}{|l|}{ Apoiante } \\
\hline M. burchellii Baker & BM, IM \\
\hline M. lundiana DC. & BM, IM \\
\hline M. microptera DC. & BA, BM, IM \\
\hline M. paranensis Dusén & $\mathrm{BM}, \mathrm{IM}$ \\
\hline M. smaragdina Dusén ex Malme & BM, IM \\
\hline M. trinervis Hook. \& Arn. & BM, IM \\
\hline M. ulei Hieron. & BM, IM \\
\hline \multicolumn{2}{|l|}{ Volúvel } \\
\hline M. campanulata Gardner & $\mathrm{BM}$ \\
\hline M. capricorni B.L. Rob. & $\mathrm{BM}$ \\
\hline M. chlorolepis Baker & BM, IM \\
\hline M. clematidifolia Dusén & $\mathrm{BM}$ \\
\hline M. cordifolia (L.f.) Willd. & $\mathrm{BM}, \mathrm{CA}$ \\
\hline $\begin{array}{l}\text { M. cynanchifolia Hook. \& Arn. ex } \\
\text { B.L. Rob. }\end{array}$ & $\mathrm{BA}, \mathrm{BM}$ \\
\hline M. dusenii B.L. Rob. & $\mathrm{BA}, \mathrm{BM}$ \\
\hline M. glomerata Spreng. & $\mathrm{BM}, \mathrm{IM}$ \\
\hline M. hastato-cordata Malme & $\mathrm{BM}$ \\
\hline M. hemisphaerica Sch. Bip. ex Baker & BM, IM \\
\hline M. hirsutissima DC. & BM, IM \\
\hline M. involucrata Hook. \& Arn. & $\mathrm{BM}$ \\
\hline M. laevigata Sch. Bip. ex Baker & BM, IM \\
\hline M. lindleyana $\mathrm{DC}$. & BM, IM \\
\hline M. micrantha Kunth & $\mathrm{BA}, \mathrm{BM}, \mathrm{IM}$ \\
\hline M. oreophila M.R. Ritter \& Miotto & $\mathrm{BM}$ \\
\hline M. orleansensis Hieron. & BM, IM \\
\hline M. parodii Cabrera & $\mathrm{BA}, \mathrm{BM}, \mathrm{CA}, \mathrm{IM}$ \\
\hline M. periplocifolia Hook. \& Arn. & $\mathrm{BA}, \mathrm{BM}, \mathrm{CA}, \mathrm{IM}$ \\
\hline M. rufescens Sch. Bip. ex Baker & $\mathrm{IM}$ \\
\hline M. salviifolia Gardner & BM, IM \\
\hline M. ternata (Vell.) B.L. Rob. & BM, IM \\
\hline M. trachypleura B.L. Rob. & $\mathrm{BM}$ \\
\hline M. variifolia Hieron. & $\mathrm{BM}$ \\
\hline M. vitifolia DC. & BM, IM \\
\hline
\end{tabular}


As onze regiões fisiográficas do Rio Grande do Sul adotadas neste estudo são as de Fortes (1959). Escolheu-se este sistema por tratar-se de classificação amplamente utilizada e por representar regiões naturais do Estado (Fig. 1): Litoral (LIT), Depressão Central (DCE), Encosta Inferior do Nordeste (EIN), Campos de Cima da Serra (CCS), Encosta Superior do Nordeste (ESN), Missões (MIS), Planalto Médio (PME), Alto Uruguai (AUR), Campanha (CAM), Serra do Sudeste (SSE) e Encosta do Sudeste (ESE).

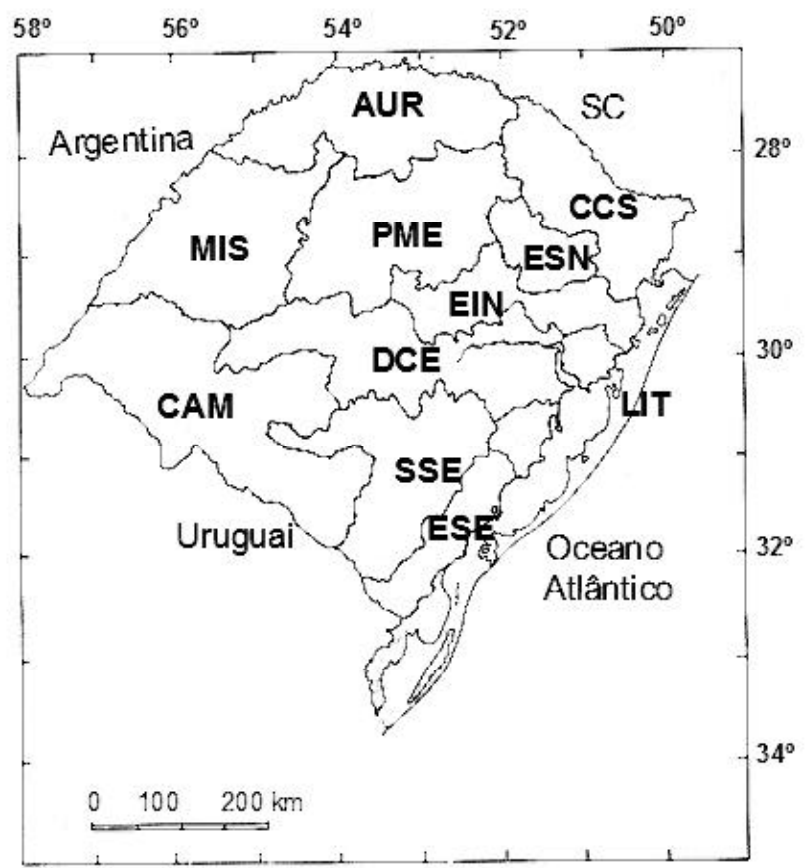

Figura 1. Regiões fisiográficas do Rio Grande do Sul, segundo Fortes (1959): Alto Uruguai (AUR), Campanha (CAM), Campos de Cima da Serra (CCS), Depressão Central (DCE), Encosta do Sudeste (ESE), Encosta Inferior do Nordeste (EIN), Encosta Superior do Nordeste (ESN), Litoral (LIT), Missões (MIS), Planalto Médio (PME), Serra do Sudeste (SSE).

Já para a América foram adotadas as onze regiões político-geográficas de Hollis \& Brummitt (1992). Esta classificação utiliza as regiões brasileiras do Centro-Oeste (BCO), Norte (BNO), Nordeste (BNE), Sudeste (BSE) e Sul (BSU). As regiões extrabrasileiras utilizadas são as seguintes: Sul da América do Sul (ASS), formada por Argentina, Chile, Paraguai e Uruguai; Oeste da América do Sul (ASW), formada por Bolívia, Colômbia, Equador, Peru; Norte da América do Sul (ASN), formada por Guiana, Guiana Francesa, Suriname e Venezuela; Mesoamérica (MES), formada por Belize, Costa Rica, El Salvador, Guatemala, Honduras, Nicarágua, Panamá, e sudeste do México; Caribe (CAR), formada por Aruba,
Bahamas, Bermuda, Cuba, Haiti, Ilhas Cayman, Jamaica, Porto Rico, República Dominicana e demais ilhas do Caribe e México (MEX), formada pelo México.

A matriz de distribuição de Mikania por regiões fisiográficas do Rio Grande do Sul foi analisada por dois métodos multivariados: uma análise de agrupamentos e uma análise de coordenadas principais, utilizando o complemento do índice de Jaccard como coeficiente de comparação (dissimilaridade) e a técnica de agrupamento soma dos quadrados. O programa de computador utilizado foi o SYN-TAX 2000 (Podani 2001).

\section{Resultados}

O número de espécies nas diferentes regiões fisiográficas do Rio Grande do Sul mostra um gradiente de riqueza decrescente entre as regiões orientais e as ocidentais (Fig. 2, Tab. 2). As regiões mais ricas foram o Litoral e a Depressão Central e as mais pobres, a Campanha e a Serra do Sudeste.

Encontrou-se espécies restritas nas regiões do Alto Uruguai (M. dusenii), dos Campos de Cima da Serra (M. oblongifolia, M. oreophila e M. smaragdina) e do Litoral (M. clematidifolia, M. lundiana e M. rufescens). Também observou-se a ocorrência de espécies em duas ou três regiões adjacentes, como Litoral e Encosta Inferior do Nordeste (M. trinervis),

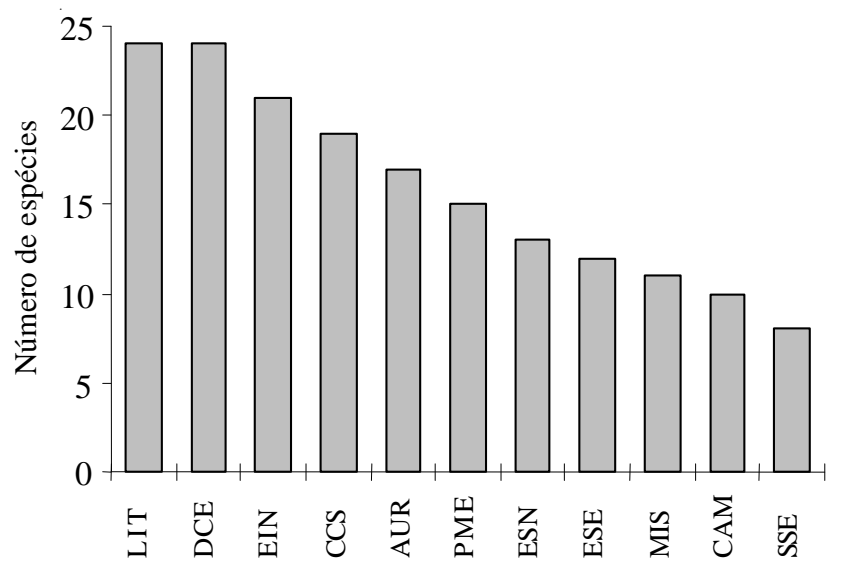

Figura 2. Número de espécies de Mikania Willd. nas diferentes regiões fisiográficas do Rio Grande do Sul, dispostas em ordem decrescente de riqueza. As regiões com os respectivos acrônimos são Litoral (LIT), Depressão Central (DCE), Encosta Inferior do Nordeste (EIN), Campos de Cima da Serra (CCS), Encosta Superior do Nordeste (ESN), Missões (MIS), Planalto Médio (PME), Alto Uruguai (AUR), Campanha (CAM), Serra do Sudeste (SSE) e Encosta do Sudeste (ESE). 
Tabela 2. Matriz de dados com a ocorrência das espécies de Mikania Willd. nas regiões fisiográficas do Rio Grande do Sul: $1=$ presente; $0=$ ausente

\begin{tabular}{|c|c|c|c|c|c|c|c|c|c|c|c|c|}
\hline Espécie & LIT & DCE & EIN & CCS & AUR & PME & ESN & ESE & MIS & CAM & SSE & Amplitude \\
\hline M. anethifolia & 0 & 1 & 0 & 0 & 0 & 0 & 0 & 0 & 1 & 1 & 0 & 3 \\
\hline M. burchellii & 0 & 0 & 1 & 1 & 1 & 1 & 1 & 0 & 1 & 0 & 0 & 6 \\
\hline M. campanulata & 1 & 1 & 1 & 1 & 0 & 0 & 1 & 1 & 0 & 0 & 0 & 6 \\
\hline M. capricorni & 0 & 1 & 0 & 1 & 1 & 0 & 0 & 0 & 0 & 1 & 0 & 4 \\
\hline M. chlorolepis & 1 & 0 & 0 & 0 & 1 & 1 & 0 & 0 & 0 & 0 & 0 & 3 \\
\hline M. clematidifolia & 1 & 0 & 0 & 0 & 0 & 0 & 0 & 0 & 0 & 0 & 0 & 1 \\
\hline M. cordifolia & 1 & 1 & 1 & 1 & 1 & 1 & 1 & 1 & 1 & 1 & 1 & 11 \\
\hline M. cynanchifolia & 1 & 1 & 0 & 0 & 0 & 1 & 0 & 1 & 0 & 1 & 0 & 5 \\
\hline M. decumbens & 0 & 0 & 1 & 1 & 0 & 0 & 0 & 0 & 0 & 0 & 0 & 2 \\
\hline M. dusenii & 0 & 0 & 0 & 0 & 1 & 0 & 0 & 0 & 0 & 0 & 0 & 1 \\
\hline M. fulva & 0 & 1 & 0 & 1 & 1 & 1 & 1 & 1 & 1 & 0 & 1 & 8 \\
\hline M. glomerata & 1 & 1 & 1 & 1 & 1 & 0 & 0 & 1 & 0 & 0 & 1 & 7 \\
\hline M. hastato-cordata & 1 & 1 & 0 & 0 & 0 & 0 & 0 & 1 & 0 & 0 & 0 & 3 \\
\hline M. hemisphaerica & 0 & 0 & 0 & 0 & 1 & 1 & 0 & 0 & 0 & 0 & 0 & 2 \\
\hline M. hirsutissima & 0 & 1 & 1 & 1 & 1 & 1 & 1 & 0 & 1 & 0 & 0 & 7 \\
\hline M. involucrata & 1 & 1 & 1 & 1 & 1 & 1 & 1 & 1 & 1 & 1 & 1 & 11 \\
\hline M. laevigata & 1 & 1 & 1 & 0 & 1 & 1 & 0 & 1 & 0 & 0 & 0 & 6 \\
\hline M. lindleyana & 1 & 0 & 1 & 0 & 1 & 0 & 0 & 0 & 0 & 0 & 0 & 3 \\
\hline M. lundiana & 1 & 0 & 0 & 0 & 0 & 0 & 0 & 0 & 0 & 0 & 0 & 1 \\
\hline M. micrantha & 1 & 1 & 1 & 1 & 1 & 1 & 1 & 1 & 1 & 1 & 1 & 11 \\
\hline M. microptera & 1 & 1 & 1 & 0 & 0 & 0 & 0 & 0 & 0 & 0 & 0 & 3 \\
\hline M. oblongifolia & 0 & 0 & 0 & 1 & 0 & 0 & 0 & 0 & 0 & 0 & 0 & 1 \\
\hline M. oreophila & 0 & 0 & 0 & 1 & 0 & 0 & 0 & 0 & 0 & 0 & 0 & 1 \\
\hline M. orleansensis & 1 & 1 & 1 & 1 & 0 & 0 & 1 & 0 & 0 & 0 & 0 & 5 \\
\hline M. paranensis & 0 & 0 & 1 & 1 & 0 & 0 & 0 & 0 & 0 & 0 & 0 & 2 \\
\hline M. parodii & 1 & 1 & 0 & 1 & 0 & 0 & 1 & 0 & 0 & 0 & 0 & 4 \\
\hline M. periplocifolia & 1 & 1 & 0 & 0 & 0 & 0 & 0 & 1 & 0 & 1 & 1 & 5 \\
\hline M. pinnatiloba & 1 & 1 & 1 & 1 & 0 & 1 & 1 & 0 & 0 & 0 & 0 & 6 \\
\hline M. rufescens & 1 & 0 & 0 & 0 & 0 & 0 & 0 & 0 & 0 & 0 & 0 & 1 \\
\hline M. salviifolia & 1 & 1 & 1 & 0 & 0 & 0 & 1 & 0 & 0 & 0 & 0 & 4 \\
\hline M. smaragdina & 0 & 0 & 0 & 1 & 0 & 0 & 0 & 0 & 0 & 0 & 0 & 1 \\
\hline M. ternata & 1 & 1 & 1 & 1 & 1 & 1 & 1 & 1 & 1 & 0 & 1 & 10 \\
\hline M. thapsoides & 0 & 1 & 0 & 0 & 1 & 1 & 0 & 0 & 1 & 1 & 1 & 6 \\
\hline M. trachypleura & 0 & 1 & 1 & 1 & 1 & 1 & 0 & 0 & 1 & 1 & 0 & 7 \\
\hline M. trinervis & 1 & 0 & 1 & 0 & 0 & 0 & 0 & 0 & 0 & 0 & 0 & 2 \\
\hline M. ulei & 1 & 1 & 1 & 0 & 0 & 0 & 0 & 0 & 0 & 0 & 0 & 3 \\
\hline M. variifolia & 1 & 1 & 0 & 0 & 0 & 1 & 0 & 0 & 1 & 1 & 0 & 5 \\
\hline M. viminea & 0 & 1 & 1 & 0 & 0 & 0 & 0 & 1 & 0 & 0 & 0 & 3 \\
\hline M. vitifolia & 1 & 0 & 1 & 0 & 1 & 0 & 1 & 0 & 0 & 0 & 0 & 4 \\
\hline Riqueza & 24 & 24 & 21 & 19 & 17 & 15 & 13 & 12 & 11 & 10 & 8 & 174 \\
\hline
\end{tabular}

Litoral (LIT), Depressão Central (DCE), Encosta Inferior do Nordeste (EIN), Campos de Cima da Serra (CCS), Alto Uruguai (AUR), Planalto Médio (PME), Encosta Superior do Nordeste (ESN), Encosta do Sudeste (ESE), Missões (MIS), Campanha (CAM), Serra do Sudeste (SSE).

Litoral, Depressão Central e Encosta Inferior do Nordeste (M. microptera e M. ulei) e Alto Uruguai e Planalto Médio (M. hemisphaerica). Espécies como $M$. cordifolia, $M$. involucrata e $M$. micrantha foram encontradas em todas as regiões fisiográficas do Rio Grande do Sul. M. ternata ocorreu em dez das regiões fisiográficas, não tendo sido encontrada até o momento na Campanha (Tab. 2). As regiões da Serra do Sudeste e Encosta do Sudeste não apresentaram espécies exclusivas. Mikania variifolia foi encontrada nas regiões da Campanha, Depressão Central, Litoral e Missões no Rio Grande do Sul.

Baseado nos dados de distribuição das espécies de Mikania no Rio Grande do Sul, o dendrograma obtido a partir da análise de agrupamentos (Fig. 3) revelou a formação de dois grandes grupos biogeográficos, um grupo oriental e outro ocidental. $\mathrm{O}$ grupo oriental dividiu-se em dois subgrupos, um de 


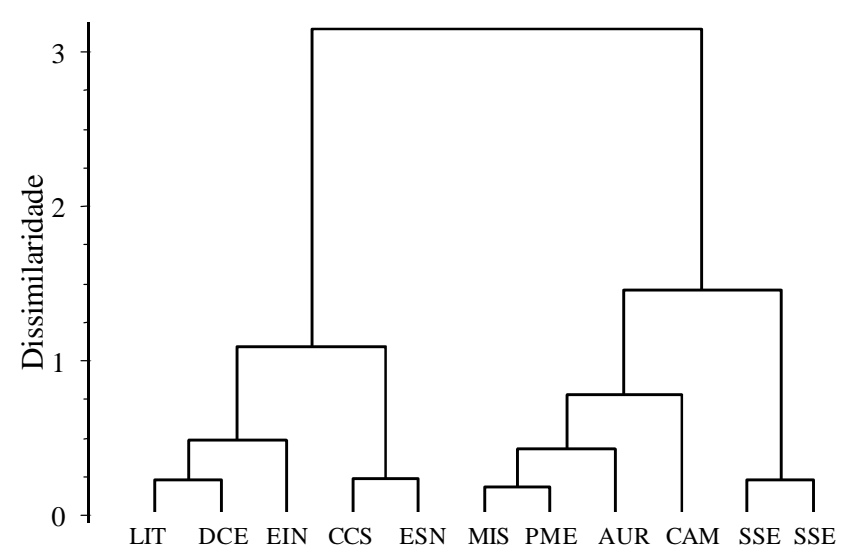

Figura 3. Relações entre regiões fisiográficas do Rio Grande do Sul, com base na distribuição de espécies de Mikania Willd. As regiões com os respectivos acrônimos são Litoral (LIT), Depressão Central (DCE), Encosta Inferior do Nordeste (EIN), Campos de Cima da Serra (CCS) e Encosta Superior do Nordeste (ESN), formando um grupo oriental; e Missões (MIS), Planalto Médio (PME), Alto Uruguai (AUR), Campanha (CAM), Serra do Sudeste (SSE) e Encosta do Sudeste (ESE), formando um grupo ocidental.

terras altas ou montano, que abrangeu as regiões fisiográficas dos Campos de Cima da Serra e Encosta Superior do Nordeste e o outro de terras mais baixas, que abrangeu as regiões fisiográficas da Encosta Inferior do Nordeste, Depressão Central e Litoral. O grupo ocidental dividiu-se em dois subgrupos, um que abrangeu as regiões da Encosta do Sudeste e Serra do Sudeste, e o outro abrangeu as regiões do Alto Uruguai, Campanha, Missões e Planalto Médio.

Nas regiões do grupo oriental o número total de táxons foi de 37, com dez restritas (o subgrupo montano apresentou 21 espécies, com quatro restritas e o subgrupo de terras baixas apresentou 34, com seis restritas). No grupo ocidental o número total de táxons foi de 25 , com duas restritas. O subgrupo das áreas basálticas apresentou 22 espécies, com duas restritas e o subgrupo do escudo granítico, apresentou 13, e não se encontrou espécies restritas (Fig. 4).

Foram encontrados quatro padrões de ocorrência das espécies de Mikania no Rio Grande do Sul, coincidentes com características climáticas e geológicas do Estado. O primeiro padrão, denominado de terras altas ou montano, tendo $M$. decumbens, M. oblongifolia, M. oreophila, M. paranensis e M. smaragdina como representantes. O segundo padrão, denominado de terras baixas, tendo como representantes $M$. campanulata, M. clematidifolia, $M$. lindleyana, M. lundiana, M. microptera, M. orleansensis, M. parodii, M. pinnatiloba, M. rufescens, $M$. salviifolia, $M$. trinervis, $M$. ulei e
M. vitifolia. O terceiro padrão, denominado padrão ocidental, englobou as espécies ocorrentes na área mais ocidental do Estado, tendo como representantes M. anethifolia, M. chlorolepis, M. capricorni, $M$. cynanchifolia, M. dusenii, $M$. glomerata, $M$. hastato-cordata, M. hemisphaerica, M. laevigata, M. periplocifolia, M. thapsoides, $M$. variifolia e M. viminea. O quarto padrão, denominado padrão amplo, englobou as espécies ocorrentes em todas ou quase todas as regiões do Estado, tendo como representantes $M$. burchellii, $M$. cordifolia, M. fulva, M. hirsutissima, M. involucrata, M. micrantha, M. ternata e M. trachypleura.

As espécies eretas (M. anethifolia, M. fulva e M. thapsoides) predominaram nas regiões campestres ocidentais, com extensões na direção oriental. Já $M$. pinnatiloba e $M$. viminea ocorreram nos campos das regiões mais orientais, enquanto que M. oblongifolia ocorreu apenas nos campos de altitude. Uma espécie volúvel, $M$. oreophila, e uma apoiante, M. smaragdina, são restritas a regiões florestais de altitude.

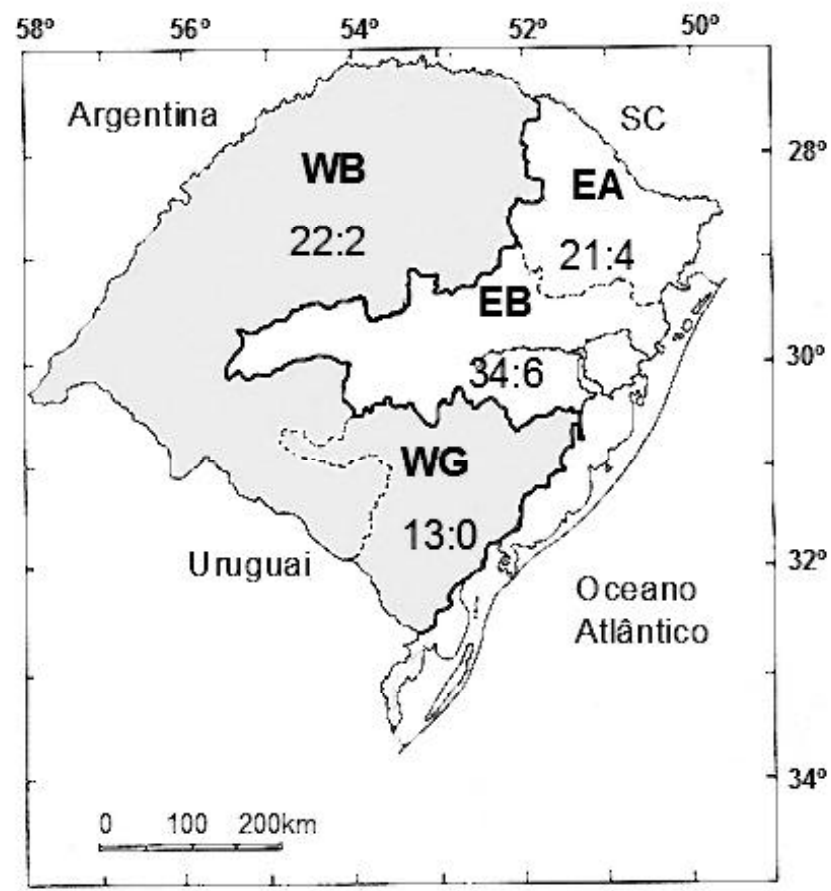

Figura 4. Regiões fisiográficas evidenciadas pelo dendrograma de similaridade entre as regiões fisiográficas do Rio Grande do Sul, baseadas na distribuição de Mikania Willd. EA: região oriental de terras altas; EB: região oriental de terras baixas; WB: região ocidental basáltica; WG: região ocidental granítica. O primeiro número representa o número total de espécies e o segundo, o número de espécies restritas. 
A análise de coordenadas principais (Fig. 5) praticamente mostrou os mesmos resultados da análise de agrupamentos, porém evidenciou melhor os dois principais fatores de distribuição de Mikania no Rio Grande do Sul. O eixo 1 representa a continentalidade, deixando as regiões orientais à direita da figura e as regiões ocidentais à esquerda.

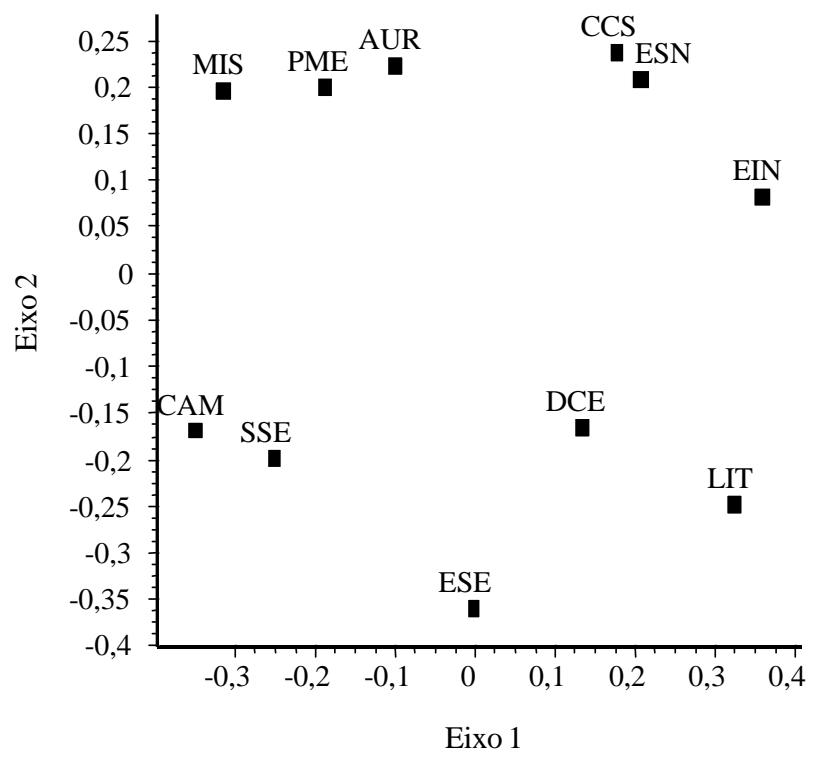

Figura 5. Relações entre regiões fisiográficas do Rio Grande do Sul, com base na distribuição de espécies de Mikania Wild., utilizando análise de coordenadas principais. O eixo 1 explica $21 \%$ e eixo 2 explica $16 \%$. As regiões com os respectivos acrônimos são Litoral (LIT), Depressão Central (DCE), Encosta Inferior do Nordeste (EIN), Campos de Cima da Serra (CCS), Encosta Superior do Nordeste (ESN), Missões (MIS), Planalto Médio (PME), Alto Uruguai (AUR), Campanha (CAM), Serra do Sudeste (SSE) e Encosta do Sudeste (ESE).
O eixo 2 representa a latitude, plotando as regiões setentrionais na parte superior e as regiões meridionais na parte inferior do gráfico. A explicação dos dois eixos atingiu $37 \%$.

O número de espécies nas diferentes regiões político-geográficas da América (Fig. 6, Tab. 3) mostrou basicamente maior ocorrência das espécies de Mikania sul-rio-grandenses na região Sul. Duas regiões adjacentes ao Rio Grande do Sul ainda apresentaram riqueza relativamente alta, o Sudeste e o Sul da América do Sul, contrastando com grande diminuição de espécies em regiões gradativamente mais distantes.

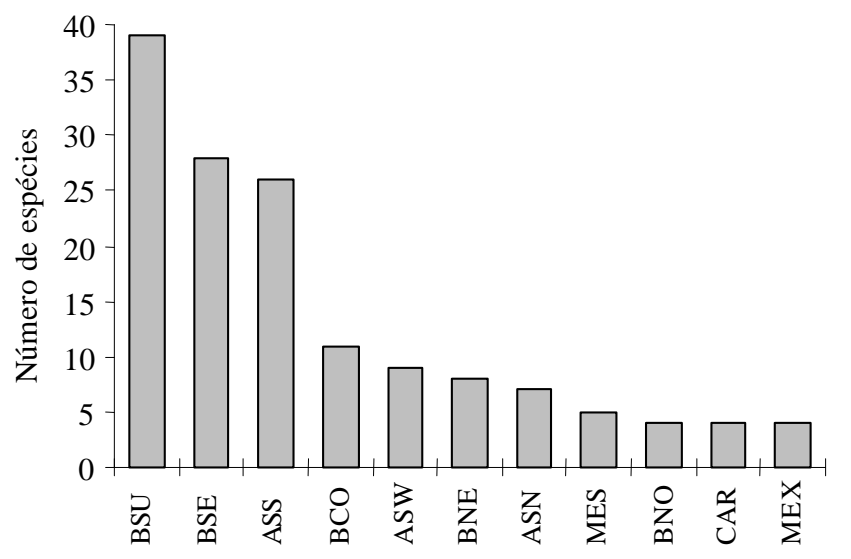

Figura 6. Número de espécies sul-rio-grandenses de Mikania Wild. nas diferentes regiões político-geográficas da América do Sul, dispostas em ordem decrescente de riqueza. As regiões com os respectivos acrônimos são Brasil Norte (BNO), Brasil Nordeste (BNE), Brasil Centro-Oeste (BCO), Brasil Sudeste (BSE), Brasil Sul (BSU), América do Sul Sul (ASS), América do Sul Oeste (ASW), América do Sul Norte (ASN), Mesoamérica (MES), Caribe (CAR), México (MEX).

Tabela 3. Matriz de dados com a ocorrência das espécies de Mikania Wild. nas regiões político-geográficas da América: 1 = presente; $0=$ ausente.

\begin{tabular}{|c|c|c|c|c|c|c|c|c|c|c|c|c|}
\hline Espécie & $\mathrm{BNO}$ & BNE & $\mathrm{BCO}$ & BSE & BSU & ASS & ASW & ASN & MES & CAR & MEX & Amplitude \\
\hline M. anethifolia & 0 & 0 & 0 & 0 & 1 & 1 & 0 & 0 & 0 & 0 & 0 & 2 \\
\hline M. burchellii & 0 & 0 & 0 & 1 & 1 & 1 & 0 & 0 & 0 & 0 & 0 & 3 \\
\hline M. campanulata & 0 & 0 & 0 & 1 & 1 & 1 & 0 & 0 & 0 & 0 & 0 & 3 \\
\hline M. capricorni & 0 & 0 & 0 & 1 & 1 & 1 & 0 & 0 & 0 & 0 & 0 & 3 \\
\hline M. chlorolepis & 0 & 0 & 1 & 1 & 1 & 1 & 1 & 1 & 0 & 0 & 0 & 6 \\
\hline M. clematidifolia & 0 & 0 & 0 & 0 & 1 & 0 & 0 & 0 & 0 & 0 & 0 & 1 \\
\hline M. cordifolia & 1 & 1 & 1 & 1 & 1 & 1 & 1 & 1 & 1 & 1 & 1 & 11 \\
\hline M. cynanchifolia & 0 & 0 & 1 & 1 & 1 & 1 & 1 & 1 & 1 & 1 & 1 & 9 \\
\hline M. decumbens & 0 & 0 & 1 & 1 & 1 & 0 & 0 & 0 & 0 & 0 & 0 & 3 \\
\hline M. dentata & 0 & 1 & 0 & 1 & 1 & 1 & 1 & 0 & 0 & 0 & 0 & 5 \\
\hline M. dusenii & 0 & 0 & 0 & 0 & 1 & 1 & 0 & 0 & 0 & 0 & 0 & 2 \\
\hline M. fulva & 0 & 0 & 0 & 0 & 1 & 1 & 0 & 0 & 0 & 0 & 0 & 2 \\
\hline M. glomerata & 0 & 1 & 1 & 1 & 1 & 1 & 0 & 0 & 0 & 0 & 0 & 5 \\
\hline M. hastato-cordata & 0 & 0 & 0 & 1 & 1 & 0 & 0 & 0 & 0 & 0 & 0 & 2 \\
\hline
\end{tabular}


Tabela 3 (continuação)

\begin{tabular}{|c|c|c|c|c|c|c|c|c|c|c|c|c|}
\hline Espécie & $\mathrm{BNO}$ & BNE & $\mathrm{BCO}$ & BSE & BSU & ASS & ASW & ASN & MES & CAR & MEX & Amplitude \\
\hline M. hemisphaerica & 1 & 0 & 0 & 1 & 1 & 1 & 0 & 0 & 0 & 0 & 0 & 4 \\
\hline M. hirsutissima & 0 & 0 & 1 & 1 & 1 & 1 & 0 & 0 & 0 & 0 & 0 & 4 \\
\hline M. involucrata & 0 & 0 & 0 & 1 & 1 & 1 & 0 & 0 & 0 & 0 & 0 & 3 \\
\hline M. laevigata & 0 & 0 & 0 & 1 & 1 & 0 & 0 & 0 & 0 & 0 & 0 & 2 \\
\hline M. lindleyana & 1 & 0 & 1 & 1 & 1 & 1 & 1 & 1 & 1 & 0 & 0 & 8 \\
\hline M. lundiana & 0 & 1 & 0 & 1 & 1 & 1 & 0 & 0 & 0 & 0 & 0 & 4 \\
\hline M. micrantha & 1 & 1 & 1 & 1 & 1 & 1 & 1 & 1 & 1 & 1 & 1 & 11 \\
\hline M. microptera & 0 & 1 & 0 & 1 & 1 & 0 & 1 & 1 & 0 & 0 & 0 & 5 \\
\hline M. oblongifolia & 0 & 0 & 0 & 1 & 1 & 1 & 0 & 0 & 0 & 0 & 0 & 3 \\
\hline M. oreophila & 0 & 0 & 0 & 1 & 1 & 0 & 0 & 0 & 0 & 0 & 0 & 2 \\
\hline M. orleansensis & 0 & 0 & 0 & 0 & 1 & 0 & 0 & 0 & 0 & 0 & 0 & 1 \\
\hline M. paranensis & 0 & 0 & 0 & 0 & 1 & 0 & 0 & 0 & 0 & 0 & 0 & 1 \\
\hline M. parodii & 0 & 0 & 0 & 0 & 1 & 1 & 0 & 0 & 0 & 0 & 0 & 2 \\
\hline M. periplocifolia & 0 & 0 & 0 & 0 & 1 & 1 & 1 & 0 & 0 & 0 & 0 & 3 \\
\hline M. pinnatiloba & 0 & 0 & 0 & 0 & 1 & 1 & 0 & 0 & 0 & 0 & 0 & 2 \\
\hline M. rufescens & 0 & 1 & 1 & 1 & 1 & 0 & 0 & 0 & 0 & 0 & 0 & 4 \\
\hline M. salviifolia & 0 & 0 & 1 & 1 & 1 & 1 & 0 & 0 & 0 & 0 & 0 & 4 \\
\hline M. smaragdina & 0 & 0 & 0 & 1 & 1 & 0 & 0 & 0 & 0 & 0 & 0 & 2 \\
\hline M. thapsoides & 0 & 0 & 0 & 1 & 1 & 1 & 0 & 0 & 0 & 0 & 0 & 3 \\
\hline M. trachypleura & 0 & 0 & 0 & 1 & 1 & 1 & 0 & 0 & 0 & 0 & 0 & 3 \\
\hline M. trinervis & 0 & 0 & 0 & 1 & 1 & 0 & 0 & 0 & 0 & 0 & 0 & 2 \\
\hline M. ulei & 0 & 0 & 0 & 1 & 1 & 0 & 0 & 0 & 0 & 0 & 0 & 2 \\
\hline M. variifolia & 0 & 0 & 0 & 0 & 1 & 1 & 0 & 0 & 0 & 0 & 0 & 2 \\
\hline M. viminea & 0 & 0 & 0 & 0 & 1 & 0 & 0 & 0 & 0 & 0 & 0 & 1 \\
\hline M. vitifolia & 0 & 1 & 1 & 1 & 1 & 1 & 1 & 1 & 1 & 1 & 1 & 10 \\
\hline Riqueza & 4 & 8 & 11 & 28 & 39 & 26 & 9 & 7 & 5 & 4 & 4 & 145 \\
\hline
\end{tabular}

Brasil Norte (BNO), Brasil Nordeste (BNE), Brasil Centro-Oeste (BCO), Brasil Sudeste (BSE), Brasil Sul (BSU), América do Sul Sul (ASS), América do Sul Oeste (ASW), América do Sul Norte (ASN), Mesoamérica (MES), Caribe (CAR), México (MEX).

Doze espécies não ocorreram em nenhum outro país da América, como M. clematidifolia, $M$. decumbens, M. hastato-cordata, M. laevigata, M. oreophila, M. orleansensis, M. paranensis, M. rufescens, M. smaragdina, M. trinervis, M. ulei e M. viminea.

As espécies de hábito ereto, como M. anethifolia, M. fulva, M. pinnatiloba, M. thapsoides e $M$. viminea, restringiram-se aos campos das regiões limítrofes à região Sul do Brasil. A única espécie decumbente restringiu-se aos campos do Brasil Central e Meridional. As volúveis e as apoiantes restringiramse ao sul do continente (região Sudeste e Sul do Brasil e Sul da América do Sul: M. burchellii, M. campanulata, M. capricorni, M. dusenii, $M$. involucrata, $M$. parodii, $M$. trachypleura e $M$. variifolia. As espécies mais tropicais como, $M$. chlorolepis, M. lindleyana, M. lundiana, $M$. microptera e $M$. rufescens atingiram seu limite sul no Rio Grande do Sul e regiões limítrofes.

M. microptera foi confirmada para o Brasil nas regiões Nordeste, Sudeste e Sul (nas regiões da Depressão Central, Encosta Inferior do Nordeste e Litoral). Esta espécie foi constatada também para a África.

\section{Discussão}

Os dois principais grupos, oriental e ocidental, e os subgrupos definidos pelos aspectos geomorfológicos, diferem do padrão encontrado por Mondin \& Baptista (1996), que encontraram para a tribo Mutisieae uma unidade planáltica e uma pampeana, coincidindo com as unidades biogeográficas propostas por Cabrera \& Willink (1980). O padrão apresentado por Mikania provavelmente deve-se ao fato de ser gênero predominantemente florestal e tropical, tendo suas espécies migrado principalmente pelo nordeste e pelo noroeste do Estado e daí para o sul (Rambo 1961). Além disso, a análise de Mondin \& Baptista (1996) considerou diversas áreas extra-brasileiras não incluídas no presente estudo. Somente a realização de 
mais abordagens analíticas, envolvendo outros táxons florestais e campestres, poderá confirmar ou refutar estes padrões discordantes.

Como ocorre com grupos de plantas tropicais, o número de espécies de Mikania diminui no sentido Norte-Sul no Brasil. Esta tendência também foi verificada por Cerana (com. pess.) para Mikania na Argentina e por Waechter (1998), para orquídeas epifíticas no leste da América do Sul. Segundo Rambo (1960), no Rio Grande do Sul diminui o número de espécies nas famílias tipicamente tropicais.

O maior número de espécies de Mikania do Rio Grande do Sul na sua porção oriental justifica-se pelo fato de que nestas regiões encontra-se uma área florestal com forte influência tropical, ambiente propício para grande parte das espécies do gênero, predominantemente florestais. Estas espécies tropicais migraram para o Estado pela "Porta de Torres", a estreita planície entre o Planalto Meridional e o oceano Atlântico, descrita por Rambo (1950) como importante via migratória de espécies tropicais do norte e do centro do Brasil. Na porção ocidental, o maior número de representantes do gênero também ocorreu em regiões com cobertura essencialmente florestal, como no Alto Uruguai, proporcionadas pela alta precipitação e baixa ocorrência de geadas.

Comparando-se o número de espécies de Mikania nas regiões florestais do Rio Grande do Sul, percebe-se que o maior contingente do gênero encontra-se nas porções do Estado que sofrem influência direta das duas principais rotas migratórias de plantas tropicais no Brasil meridional. Uma delas ocorreu pelo noroeste do Estado, ao longo da bacia Paraguai-Paraná-Uruguai, e outra ocorreu para o sul a partir de uma área situada entre São Paulo e Espírito Santo, no litoral atlântico (Rambo 1954). A partir do Alto Uruguai a rota teria seguido ao longo dos vales fluviais para o sul e para o leste, sendo considerada mais antiga que a do litoral (Rambo 1956). Segundo Rambo (1960) o avanço da floresta pluvial ocorre pelos rios e pelos cursos d'água, por onde várias espécies de Mikania devem ter migrado. Muitas destas espécies tropicais têm seu limite sul no Estado. Uma terceira rota migratória percorrida por espécies de Mikania ocorreu ao longo do Planalto Sul-Brasileiro, entrando no Estado através do rio Pelotas, que depois dá origem ao rio Uruguai, rota também reconhecida por Rambo (1950). As três rotas migratórias verificadas para Mikania são observadas também para o grupo das leguminosas tropicais (Rambo 1953) e temperadas (Miotto \& Waechter 1996) e para diversas espécies arbóreas (Jarenkow \& Waechter 2001).

O Rio Grande do Sul é considerado região de transição fitogeográfica, como foi observado por Rambo (1960) para a flora em geral, e, por Waechter (1998) para orquídeas epifíticas no leste da América do Sul. O paralelo $30^{\circ} \mathrm{S}$ representa uma transição florística e vegetacional marcante em toda a América do Sul (Cabrera \& Willink 1980; Waechter 2002).

O maior número de espécies restritas nas matas de terras baixas do Litoral e nos campos e matas de altitude dos Campos de Cima da Serra podem ser explicadas pela proximidade das regiões de entrada das correntes migratórias. As espécies tropicais migraram pela "Porta de Torres" e atingiram seu limite sul nesta região. As florestas encontradas nesta área caracterizam-se pela grande diversidade específica, proporcionada pela umidade e temperatura relativamente altas (Leite 2002). Nos Campos de Cima da Serra, as espécies montanas do Planalto Sul-Brasileiro ocorrem em borda ou interior de mata, com Araucaria angustifolia (Bertol.) Kuntze (como M. oreophila e M. smaragdina) ou nos campos protegidos do gado (como M. oblongifolia). Nesta área verificam-se as maiores altitudes do Estado, chegando a atingir cerca de $1.400 \mathrm{~m}$ e as menores temperaturas, com freqüente ocorrências de geadas (Leite 2002). Endemismos acarretados por estas condições particulares do clima são encontrados nesta região, como em Holocheilus monocephalus C. Mondin (Mondin 1995) e Pamphalea ramboi Cabrera (Mondin \& Baptista 1996) para Asteraceae-Mutisieae, em Adesmia reitziana Burk. (Miotto \& Leitão Filho 1993) e Lupinus uleanus C.P. Sm. (Pinheiro \& Miotto 2001) para Leguminosae-Faboideae, e em Piptochaetium alpinum L.B. Smith e Stipa brasiliensis A. Zanin \& Longhi-Wagner (Zanin et al. 1992) para PoaceaeStipeae.

O número baixo de espécies de Mikania encontrado na Serra do Sudeste e na Campanha pode ser explicado pela diminuição, no sentido norte-sul, de formações florestais, aliado ao clima mais adverso às espécies tropicais. Nestas regiões as matas resumem-se a formações de baixo porte e a matas ciliares. Por outro lado, são regiões botanicamente pouco exploradas, podendo ocorrer espécies ainda não descobertas. Rambo (1952) afirma que nas compostas sul-brasileiras há forte endemismo nas espécies meridionais campestres, inclusive em Mikania. Algumas espécies eretas, como $M$. anethifolia, M. fulva, M. pinnatiloba e M. viminea têm seu limite norte na região Sul do Brasil, não atingindo as regiões 
mais quentes do país, o que foi comprovado neste estudo.

A explicação total dos eixos da ordenação pode ser considerada relativamente baixa, indicando a importância secundária de outros fatores na distribuição regional do gênero Mikania. Como continentalidade e latitude estão correlacionados sobretudo com parâmetros climáticos, os prováveis fatores secundários devem estar relacionados a substratos geológicos, tipos de solos e de vegetação. Por exemplo, a distribuição de campos e florestas, que representa importante fator para a distribuição de Mikania no Rio Grande do Sul, não está correlacionada com os principais fatores evidenciados na análise de coordenadas principais.

$\mathrm{O}$ padrão anfiatlântico de $M$. microptera tem provocado opiniões conflitantes. Segundo Holmes \& McDaniel (1982), embora apareça com maior regularidade nas coleções africanas, aparentemente é nativa em ambos os continentes. Holmes (1982) acreditava tratar-se de uma introdução da espécie na América do Sul ou na África. King \& Robinson (1987) e, posteriormente, Holmes (1995), concordaram que aparentemente trata-se de distribuição natural. De acordo com King \& Robinson (1987), este padrão certamente é o resultado da distribuição de M. microptera por pássaros. O padrão disjunto entre o continente americano e africano ocorre em inúmeros outros gêneros, como em Jatropha (Euphorbiaceae), Machaerium (Fabaceae) e Lantana (Verbenaceae), entre outros (Waechter 2002). A distribuição anfiatlântica de M. microptera certamente representa um caso de dispersão a longa distância em tempos recentes, por se tratar de uma mesma espécie, ou seja, ainda não diferenciada ao nível supra-específico.

A única espécie considerada disjunta no continente americano é Mikania ternata, que, de acordo com Holmes (1995), tem distribuição que inclui o Sudeste do Brasil, Paraguai e Argentina, e ocorre como disjunta no Peru. No entanto, na revisão de herbários encontrou-se material da Bolívia, verificando-se, portanto, a ocorrência contínua de $M$. ternata até o norte da Argentina e Paraguai.

Segundo Cabrera \& Klein (1989), M. variifolia (citada como M. sapucayensis Hassler), teria migrado através da bacia do rio Uruguai para a bacia do rio Jacuí e daí em sentido nordeste até o extremo sudeste de Santa Catarina, seguindo uma rota migratória sulleste-nordeste. Observando-se as regiões em que esta espécie foi coletada no Rio Grande do Sul, verifica-se que $M$. variifolia realmente deve ter percorrido a rota descrita por Cabrera \& Klein (l.c.).

Considerando a distribuição na América, como era de se esperar, nas regiões limítrofes com o Rio Grande do Sul encontra-se o maior número de espécies em comum, diminuíndo a riqueza à medida que aumenta a distância do Estado. As espécies com distribuição mais ampla nas Américas, M. micrantha e M. cordifolia, também encontram-se bem distribuídas no Estado, evidenciando a grande capacidade de adaptação destas espécies a diferentes climas e hábitats. Para King \& Robinson (1987), a distribuição pantropical de $M$. micrantha parece ser influenciada pela interferência humana, uma vez que foi introduzida na Ásia.

\section{Referências bibliográficas}

Boechat, S.C. \& Longhi-Wagner, H.M. 2000. Padrões de distribuição geográfica dos táxons brasileiros de Eragrostis (Poaceae, Chloridoideae). Revista Brasileira de Botânica 23(2): 177-194.

Cabrera, A.L. \& Klein, R.M. 1989. Compostas (Eupatorieae). Flora Ilustrada Catarinense p. 649-750.

Cabrera, A.L. \& Willink, A. 1980. Biogeografia de America Latina. OEA. Washington.

Fortes, A.B. 1959. Geografia física do Rio Grande do Sul. Ed. Globo, Porto Alegre.

Hollis, H. \& Brummitt, R.K. 1992. World geographical scheme for recording plant distributions. International working group on taxonomic databases for plant cciences/Hunt Institute for Botanical Documentation Carnegie Mellon University, Pittsburg.

Holmes, W.C. 1982. Revision of the old world Mikania (Compositae). Botanische Jahrbücher für Systematik Pflanzengeschichte und Pflanzengeografh 103(2): 211-246.

Holmes, W.C. 1995. A review preparatory to an infrageneric classification of Mikania (tribe: Eupatorieae). Pp. 239-254. In: D.J.N. Hind; C. Jeffrey \& G.V. Pope (eds.). Advances in Compositae Systematics, Royal Botanical Gardens, Kew.

Holmes, W.C. 1996. A proposed sectional classification for Mikania (Eupatorieae). Pp. 621-626. In: D.J.N. Hind (ed.) Compositae: Systematics. Royal Botanical Gardens, Kew.

Holmes, W.C. \& McDaniel, S. 1982. Genus Mikania - Tribe Eupatorieae. In: J.F. Macbride. Flora of Peru. Fieldiana Bot. New Series 9: 1-57.

Jarenkow, J.A. \& Waechter, J.L. 2001. Composição, estrutura e relações florísticas do componente arbóreo de uma floresta estacional no Rio Grande do Sul, Brasil. Revista Brasileira de Botânica 24(3): 263-272.

King, R.M. \& Robinson, H. 1987. The genera of the Eupatorieae (Asteraceae). Missouri Botanical Garden, St. Louis.

Leite, P.F. 2002. Contribuição ao conhecimento fitoecológico do sul do Brasil. Ciência \& Ambiente 24: 51-73. 
Longhi-Wagner, H.M. 1990. Diversidade e distribuição geográfica das espécies de Aristida L. (Gramineae) ocorrentes no Brasil. Acta Botanica Brasilica 4: 105-124.

Longhi-Wagner, H.M. \& Zanin, A. 1998. Padrões de distribuição geográfica das espécies de Stipa L. (Poaceae-Stipeae) ocorrentes no Brasil. Revista Brasileira de Botânica 2: 167-175.

Miotto, S.T.S. \& Leitão Filho, H.F. 1993. LeguminosaeFaboideae, gênero Adesmia DC. Boletim do Instituto de Biociências, Botânica 52: 1-157.

Miotto, S.T.S. \& Waechter, J.L. 1996. Considerações fitogeográficas sobre o gênero Adesmia (LeguminosaeFaboideae) no Brasil. Boletin de la Sociedad Argentina de Botánica 32(1-2): 59-66.

Mondin, C.A. 1995. Holocheilus monocephalus (AsteraceaeMutisieae), nova espécie do sul do Brasil. Napaea 11: 31-34.

Mondin, C.A. \& Baptista, L.R.M. 1996. Relações biogeográficas da tribo Mutisieae Cass. (Asteraceae), sensu Cabrera, no Rio Grande do Sul. Comunicações do Museu de Ciências Tecnológicas da Pontifícia Universidade Católica do Rio Grande do Sul, Bot. 2(1): 49-152.

Pinheiro, M. \& Miotto, S.T.S. 2001. Leguminosae-Faboideae, gênero Lupinus L. Boletim do Instituto de Biociências, Botânica 60: 1-100.

Podani, J. 2001. SYN-TAX 2000, computer programs for analysis in ecology and systematics. Scientia Publishing, Budapest.
Rambo, B. 1950. A porta de Torres. Anais Botânicos do Herbário Barbosa Rodrigues 2: 9-20.

Rambo, B. 1952. Análise geográfica das compostas sulbrasileiras. Anais Botânicos do Herbário Barbosa Rodrigues 4: 87-160.

Rambo, B. 1953. Estudo comparativo das leguminosas riograndenses. Anais Botânicos do Herbário Barbosa Rodrigues 5: 107-184.

Rambo, B. 1954. Análise histórica da flora de Porto Alegre. Sellowia 6: 9-112.

Rambo, B. 1956. Der Regenwald am oberen Uruguay. Sellowia 7: 183-233.

Rambo, B. 1960. Die südgrenze des brasilianischen regenwaldes. Pesquisas 8: 5-41.

Rambo, B. 1961. Migration routes of the south brazilian rain forest. Pesquisas 12: 5-54.

Stuessy, T.F. \& Garver, D. 1996. The defensive role of pappus in heads of Compositae. Pp. 81-91. In: P.D.S. Caligari \& D.J.N. Hind (eds.). Compositae: biology \& utilization. Royal Botanical Gardens, Kew.

Waechter, J.L. 1998. Epiphytic orchids in eastern subtropical South America. 494p. Proccedings of the $15^{\text {th }}$ World Orchid Conference. Turriers, Naturalia Publications, Rio de Janeiro.

Waechter, J.L. 2002. Padrões geográficos na flora atual do Rio Grande do Sul. Ciência \& Ambiente 24: 93-108.

Zanin, A.; Mujica-Salles, J. \& Longhi-Wagner, H.M. 1992. Gramineae, tribo Stipeae. Boletim do Instituto de Biociências, Botânica 51: 1-174. 